Case Report,

\title{
Keratinizing Squamous Cell Carcinoma of the Palate in Clinically Negative Neck: Case Report and Review
}

\author{
Grace Paka Lubamba ${ }^{1,2}$, Peng Kun Xu ${ }^{1}$, Jian Guan ${ }^{1 *}$, Xin Yu Wang ${ }^{1}$, Shang Bo Ning ${ }^{1}$, Xin Peng Dai ${ }^{1}$, \\ Zhen Yu Song ${ }^{1}$, Jian Yu Du ${ }^{1}$, Bao Rui Ren ${ }^{1}$ \\ ${ }^{1}$ Department of Oral and Maxillofacial Surgery, Second Affiliated Hospital of Jiamusi University, Jiamusi, \\ China \\ ${ }^{2}$ Service of Oral and Maxillofacial Surgery, Department of Dental Medicine, Faculty of Medicine, \\ University of Kinshasa, Kinshasa, Democratic Republic of Congo
}

E-mail: guanjian1207@163.com

\begin{abstract}
:
Background: The Squamous cell carcinoma of the palate is rare; women are more affected than men. This study aimed to discuss the etiology, diagnostic modalities, prognosis factors, and treatment strategies.

Case: A 49-year-old woman was admitted to our department in 2019 with a chief complaint of a hard and painful mass on the right palate. The Clinical assessment revealed a palatal tumor extended to 3/4 of hard palate and part of the soft palate, with hard consistency, no mobility, cauliflower-like surface partly covered by a white pseudo-membrane, dark red-colored, and clinically negative neck. 3D CT scan of palate and maxilla revealed malignant lesion features, Cervical MRI and Chest CT revealed enlarged lymph nodes. The histopathological assessment diagnosed keratinizing squamous cell carcinoma of the right palate. Resection of the tumor and Reconstructive surgery were performed. The postoperative outcomes were simple.

Conclusion: Hepatitis B virus, local chronic inflammations were the risk factors incriminated in this case. The prognosis factors such as patient age, tumor size, lymph node involvement, bone invasion, and pathological grade influence the treatment and survival. Selective neck dissection, Ablative surgery, and Reconstruction with Submental island flap represented our management strategy for keratinizing squamous cell carcinoma of the palate.
\end{abstract}

Keywords: Neck dissection, palate, reconstructive surgical procedure, squamous cell carcinoma

\section{Interoduction:}

Squamous Cell Carcinoma (SCC) of the head and neck regions represents the sixth most common cancer in male patients after lung, prostate, colorectal, stomach, and bladder cancer; but the tenth most common cancer in female patients after breast, colorectal, lung, stomach, uterus, cervix, ovary, bladder and liver cancer [1-4]. It is usually encountered during the sixth and seventh decades of life, but the increase of patients under the fifth decade was recently reported in the literature [3]. Squamous Cell Carcinoma (SCC) is considered the most common malignant tumor of the oral cavity [1,5-7] that represents approximately $90-95 \%$ of all oral malignant tumors [4,7]. At least half of the head and neck malignant tumors reported in the literature are oral SCC [1]. The SCC of the palate as well as SCC of maxillary gingiva and maxillary alveolus are relatively rare compared to other sites of oral SCC $[1,3,5,6,8]$. SCC accounts for $66 \%$ of malignant tumors of the hard palate and maxillary alveolus. One of the main reasons for the rarity of SCC of the palate is the fact that it is usually ulcerative and maxilla bone invasion occurs early [5].

The SCC can arise from malignant transformation of a previously existing potentially malignant lesion not treated or not well treated such as oral leukoplakia, erythroplakia, submucous fibrosis, and lichenoid dysplastic; it can arise also de novo [4]. The two common risk factors involved in the etiology of oral SCC are lifestyle factors such as 
excessive alcohol drinking and tobacco smoking. Other etiologic factors include immune deficiency, genetic factors, dietary factors, some viral infections, sunlight exposure, ionizing radiation [24]. Because the lesion is painless in the majority of cases, oral SCC and its precursor lesions are often discovered late, most of the time as an incidental finding during a routine dental or medical examination [2]. SCC of palate and SCC of maxilla have anatomic proximity, similar presentation, oncologic behavior, and outcomes; but represent two different entities [1,6]. The patient's age, the cervical lymph node involvement, the time of diagnosis, the pathological grade, and the size of the tumor are the most important and major prognosis factors influencing treatment planning and survival $[2,5,9]$.

The published data related to SCC of the palate are rare, particularly concerning the management of the clinically negative neck $\left(\mathrm{N}_{0}\right)$, the reconstructive surgical procedures of the resulting defect after ablative surgery, and treatment outcomes $[1,8]$. In this report, we have presented a case of keratinizing squamous cell carcinoma of the right palate (well-differentiated) in clinically negative $\left(\mathrm{N}_{0}\right)$ neck. We have discussed the etiology, diagnostic modalities including clinical, imaging, and histopathological assessment, as well as the prognosis factors and treatment strategies.

\section{Case presentation:}

A 49-year-old woman was admitted to the outpatient clinic of our department for the first time in 2018 with a chief complaint of soft and painless mass on the right hard palate, accompanied by toothache, and gradually enlarged. The clinical assessment revealed a mass presenting the aspect of cauliflower-like surface ulcerated. There was no history of tobacco smoking and alcohol drinking. The oral hygiene was poor. A biopsy of the lesion was performed and the specimen was sent to a histopathological laboratory for analysis. The pathological report diagnosed gingival squamous papillary hyperplasia with chronic inflammation (Figure 1A and 1B), and suggested a close observation of the lesion area after treatment. Surgical treatment was recommended to remove the mass and altered mucosa and prevent a possible malignant transformation of the lesion, but the patient did not agree with the suggested treatment plan. During the disease, the patient had taken traditional medicine and anti-inflammatory medicine by herself, and the mass subsided, the color of the mucosa changed slightly from purple to red. At the end of 2018, she was hospitalized in an external hospital, where another biopsy was performed and the pathological diagnosis was also an inflammatory lesion. The patient reported that the mass decreased and she felt better for a while. A few months later the mass grew rapidly and became painful, affecting eating.

In 2019 the patient was admitted to our department for the second time, almost 2 years since she discovered the lesion. After clinical assessment, there were some clinical findings of the first consultation with additional features (see below) (Figures 2A and 2B). Preoperative examinations were composed by Hematological assessment with tests of Hepatitis B Virus (HBV) and Human Immunodeficiency Virus (HIV), 3D CT scan of the palate and maxillary (Figure 3), Cervical MRI (Figure 4), Chest CT (Figure 5), Biopsy of the lesion for histopathological analysis, and Systematic Review of the patient through a collaborative approach with other medical specialties.

There was no obvious abnormality in the systematic review of the patient, the general condition was normal. A slightly enlarged lymph node was palpable in the right submandibular area. The lymph node has good mobility; it was around $1 \mathrm{~cm}$ in size and painless. There was no obvious abnormality in the degree of mouth opening and the opening pattern. The palatal tumor extended to $3 / 4$ of the hard palate and part of the soft palate. The palatal tumor protruding surface was about $1 \mathrm{~cm}$, with hard consistency, no mobility, cauliflower-like surface, dark red color, the surface was partly covered by a white pseudo-membrane, with unclear boundaries, no numbness in the palate, severe tenderness, the pain was intense. The teeth 15,16 , and 17 were missing. During the supplementary examination, the test of Hepatitis B infection revealed the following features: Hepatitis $B$ virus surface antigen: $0.05 \mathrm{ng} / \mathrm{ml}$, Hepatitis B virus surface antibody: $12.32 \mathrm{mIU} / \mathrm{ml}$, Hepatitis B virus core antibody: $60 \mathrm{PEIU} / \mathrm{ml}$.

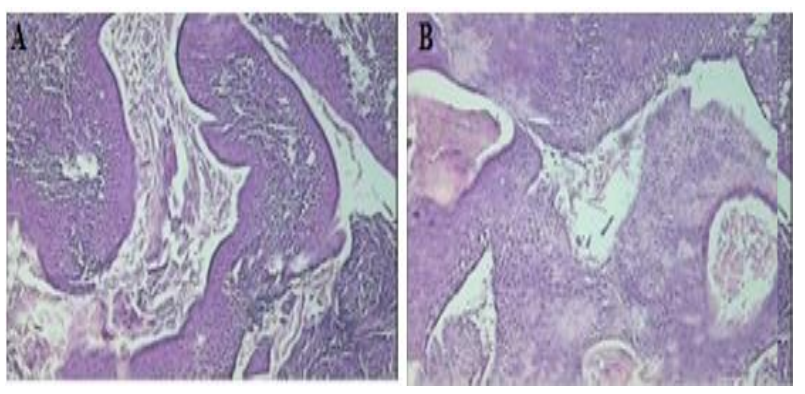


Figure1. (A) and (B): Histopathological images of Squamous papillary hyperplasia with chronic inflammation of the right palatal mucosa. Formalin fixed a pile of gray red-gray brown tissue, $2.7 \mathrm{~cm} \times 1.6 \mathrm{~cm} \times 0.4 \mathrm{~cm}$ (magnification: $10 x$ and $40 x$ ));

The clinical and imaging findings have led to a diagnosis of carcinoma of the right palate, most likely a squamous cell carcinoma. The differential diagnosis was made with Maxillary myofibroma and Giant cell granuloma of the maxilla. The pathological report diagnosed a Keratinizing squamous cell carcinoma of the right palate, highly differentiated, with local interstitial infiltration (Figure 6). The specimen was fixed in $10 \%$ buffered formalin and embedded in paraffin. Visual examination: there were 5 pieces of the grayish red non-plastic tumor, with the size from $0.5 \times 0.3 \times 0.3 \mathrm{~cm}$ to $1.5 \times 0.3 \times 0.5 \mathrm{~cm}$, with a cauliflower-like aspect on the surface, firm, graywhite, and medium in quality. The treatment strategy consisted of a selective neck dissection; surgical extended resection of the tumor by right subtotal maxillectomy and left partial maxillectomy; and reconstruction of the defect with Submental Island Flap (SIF).
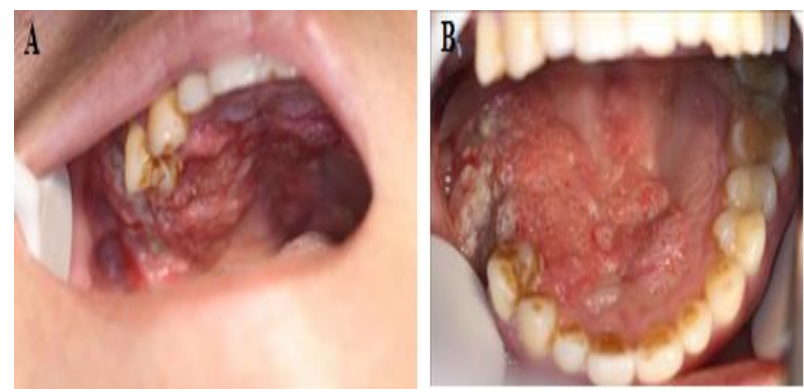

Figure2. Preoperative clinical aspect of the lesion in direct view $(A)$ and indirect view by a mirror $(B)$.

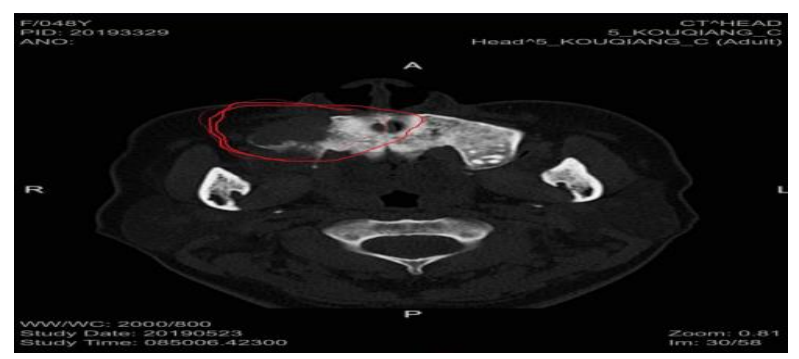

Figure3. 3D CT scan of palate and maxillary showing irregular soft tissue density mass and boundary on the right palatal mucosa with adjacent osteolytic destruction, considered as a malignant lesion.

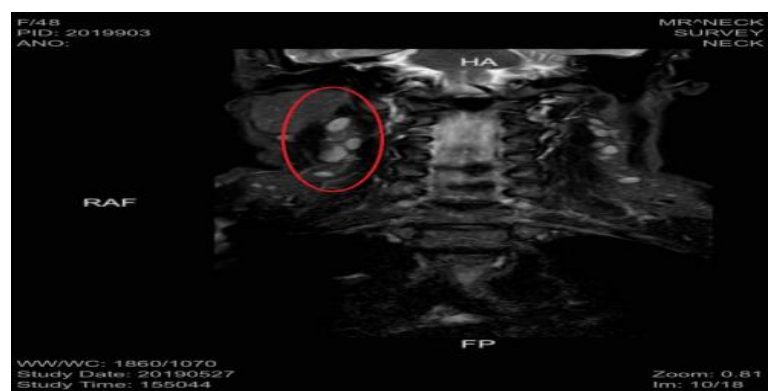

Figure4. Cervical MRI revealing multiple enlarged lymph nodes in the bilateral submandibular space and the bilateral neck. It was doubtful that metastatic tumors in lymph nodes were found, but there was a suspicion of occult metastasis.

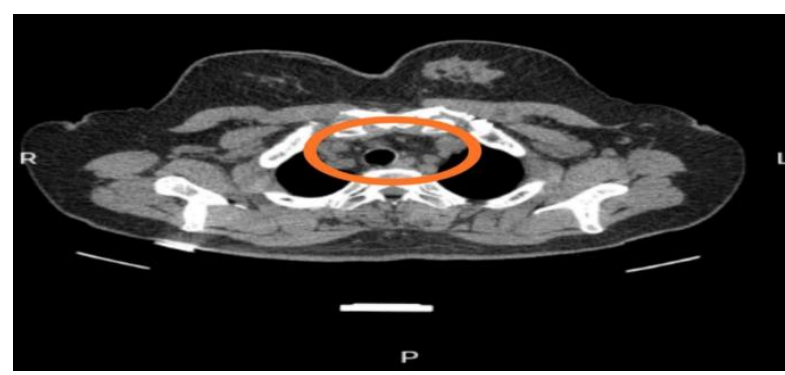

Figure5. Chest $C T$ revealing multiple enlarged lymph nodes in the mediastinum (mediastinal lymphadenopathy) with thickening of the left pleura, no obvious metastases were found in the lungs.

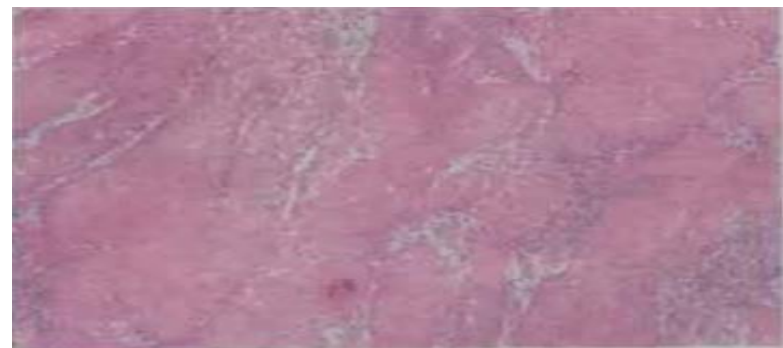

Figure6. Preoperative histopathological image of Keratinizing squamous cell carcinoma of the right palate well-differentiated, with local interstitial infiltration (magnification: 40x)

\section{Discussion:}

SCC of the palate is often encountered in women than men compared to other sites of oral SCC such as the tongue and floor of the mouth; the bone invasion can occur much earlier because the soft tissue barrier is thin [1,5]. Many studies have reported an increased incidence of oral SCC in young adults under the fifth decade. In their study, Cariati et al [3] revealed that more than half of the patients were in the fourth decade and under the fourth decade. In the present study, we reported a case of a fourth-decade patient, especially a 49year-old woman.

The current hypothesis about carcinogenesis of 
oral SCC consists of an accumulation of genetic mutations in the oral epithelial cells, which may be favored by some risk factors such as excessive alcohol drinking and tobacco smoking [3,10]. However, there is an emerging population of patients with oral malignant tumors, but without exposure or with slight exposure to these two common risk factors, especially young and elderly women $[10,11]$. This raises the possibility of involvement of other risk factors such as immune deficiency, genetic factors, dietary factors, viruses, local chronic inflammations or infections, etc. [3]. Concerning the genetic factors of oral SCC, investigations are needed to determine the role of some predisposing genetic disorders such as Fanconi and dyskeratosis congenita. Concerning the viral etiology, it was reported that $12 \%$ of oral malignant tumors are caused by one of the following seven well known oncogenic viruses: Hepatitis B and C Viruses (HBV, HCV); Human T-Lymphotropic Virus 1 (HTLV-1); Human Papilloma Virus (HPV); Kaposi's sarcomaassociated Herpes Virus (HHV-8); Merkel Cell Polyomavirus (MCPyV); and Epstein-Barr virus (EBV). Concerning the local chronic inflammations and infections, it is widely accepted that they may induce changes in oral microbiota that would increase the risk of oral malignant tumors [11].

In this case, there was no history of alcohol drinking and tobacco smoking. The viral origin could be suspected as one of the main risk factors because the serologic test of Hepatitis B-virus revealed the following results: Hepatitis $B$ virus surface Antigen (HBsAg): 0.05ng/ml, Hepatitis B virus surface Antibody (HBsAb): $12.32 \mathrm{mIU} / \mathrm{ml}$, Hepatitis B virus core Antibody (HBcAb): 60 PEIU/ ml. The normal limits considered were HBsAg: 0-0.2 ng/ml, HBsAb: $0-10 \mathrm{mIU} / \mathrm{ml}$, $\mathrm{HBcAb}$ : 0-15 PEIU/ ml. The local chronic inflammation, especially squamous papillary hyperplasia with chronic inflammation of the right palatal mucosa could be also suspected as another risk factor for this case. The fact that the surface of the lesion was partly covered by a white pseudomembrane could also suspect the previous presence of a potentially malignant lesion, especially leukoplakia. The mechanisms that may explain the role of $\mathrm{HBV}$ in oral carcinogenesis remain unknown, but it is believed that the virus may play a role in both direct and indirect oncogenic mechanisms [12].

According to Dona et al [13], the findings of their study were clinically relevant and have added evidence that $\mathrm{HBV}$ and $\mathrm{HCV}$ infections are associated with SCC. Many studies are revealing an increasing incidence of oral SCC among HBsAg seropositive patients [13-15]. In the study of Komori et al [12], head and neck SCC and head and neck Adenocarcinoma diagnosis were correlated with Hepatitis B virus core Antibody $(\mathrm{HBc} A b)$ positivity. In this case, the suspicion of $\mathrm{HBV}$ as a risk factor was based on the $\mathrm{HBcAb}$ positivity, because the patient was $\mathrm{HBsAg}$ seronegative.

The slightly enlarged lymph node palped in the right submandibular area has good mobility; it was around $1 \mathrm{~cm}$ in size and painless. This was considered a clinically negative neck $\left(\mathrm{N}_{0}\right)$ because of the lack of evidence of malignity characteristics and the size of enlargement that was almost similar to the normal limit in size (inferior or equal to 1 $\mathrm{cm})$. However, the possibility of occult metastases has not been overlooked. The clinical TNMstaging was $\mathrm{T}_{4} \mathrm{~N}_{0} \mathrm{M}_{\mathrm{x}}$.

SCC of palate often invades maxillary bone early, to understand the degree and extent of maxillary bone destruction, a CT scan of palate and maxilla was performed, which revealed irregular soft tissue density mass and boundary on the right palatal mucosa with adjacent osteolytic destruction, considered as malignant lesion features. Because metastasis of SCC of the palate is mainly to the deep superior cervical lymph nodes, the cervical MRI was performed, which revealed multiple enlarged lymph nodes in the bilateral submandibular space and the bilateral neck. There was a doubt about the presence of metastatic tumors in lymph nodes and suspicion of occult metastasis. Because distant metastasis of oral SCC is often to the lungs, a chest CT was performed, which revealed multiple enlarged lymph nodes in the mediastinum (mediastinal lymphadenopathy) with thickening of the left pleura, no obvious metastases were found in the lungs. Despite the technological progress made on imaging, there is no available imaging technique that can detect the presence of micrometastases [5].

There is an association between tumor site, size, and grade with the risk of cervical lymph node metastasis for oral SCC of any site including SCC of palate [2]. The factors representing a poor prognosis include the advanced age, large tumor size, presence of cervical lymph nodes metastases, the deep invasive front of the tumor (deep infiltration of the submucosa and deep bone 

Report and Review

invasion), and the loss of differentiation characterized by undifferentiated and poorly differentiated SCC [4,7].

According to the degree of differentiation of the neoplastic cells, the oral SCC is divided into 4 grades: $\mathrm{G}_{0}$ : Undifferentiated oral $\mathrm{SCC} ; \mathrm{G}_{1}$ : Highly or Well-differentiated oral SCC; $\mathrm{G}_{2}$ : Moderately differentiated oral SCC; and $\mathrm{G}_{3}$ : Poorly differentiated oral SCC. The grade $\mathrm{G}_{3}$ has the worst prognosis. The grades $\mathrm{G}_{0}$ and $\mathrm{G}_{3}$ have a poor prognosis compared to $G_{1}$ and $G_{2}$ [10]. $G_{1}$ oral SCC and $\mathrm{G}_{2}$ oral SCC can be grouped as low-grade tumors; $\mathrm{G}_{0}$ oral SCC and $\mathrm{G}_{3}$ oral SCC as highgrade tumors [4]. Proper identification of the histological variant and grade of oral SCC in presence is an important factor for better treatment planning [7]. We reported a case of welldifferentiated oral SCC, especially a keratinizing squamous cell carcinoma of the right palate, which was considered as low grade and presented a good prognosis.

It was reported in the literature that oral SCC in the midline or exceeding this line are predisposed for contralateral or bilateral cervical lymph nodes metastasis because of the crossing lymphatic vessels in the midline. Also, the cervical lymph nodes metastasis is more often present in postcanine SCC, but when there is a cervical lymph nodes metastasis in a pre-canine SCC, the probability of bilateral cervical lymph nodes metastasis is high compared to post-canine SCC [2].

Concerning the treatment strategy, there are generally 3 categories of factors that may influence the choice of treatment of SCC of the palate: tumor factors, patient factors, and physician factors [16]. *Tumor factors include size (T-stage), location, bone invasion, presence or absence of cervical lymph nodes involvement, type of previous treatment, and histopathological features (type, grade, degree of invasion) [7,16]. *Patient factors include age, general medical condition (assessed during patient systematic review in a collaborative approach with other medical specialties), tolerance of treatment, professional occupation, acceptance and compliance, lifestyle (mainly tobacco smoking and alcohol drinking), and socio-economic considerations $[10,16]$. *Physician factors include technical equipment availability, expertise from various disciplines through a multidisciplinary approach including surgery (ablative and reconstructive), oncology (radiotherapy, chemotherapy), prosthodontics, implantology, psychology (psycho-social support), etc. [16].

The surgical treatment strategies included the following procedures: Intraoperative design of flap size 5 × $9 \mathrm{~cm}$ (Figure 7A); Submental island flap raising (Figure 7B) with ipsilateral supraomohyoidal neck dissection (levels I, II, III, and $\mathrm{Va}$ on the right side) (Figure 8A) and partial contralateral selective neck dissection of levels I and IIa; Extended resection of SCC of palate through a right subtotal maxillectomy and left partial maxillectomy (Figures 8B; Figures 9A and 9B); Reconstruction of the remaining defect with SIF transfer and fixation (Figures 10A and 10B; Figure 11A); and Tracheostomy (Figure 11B).
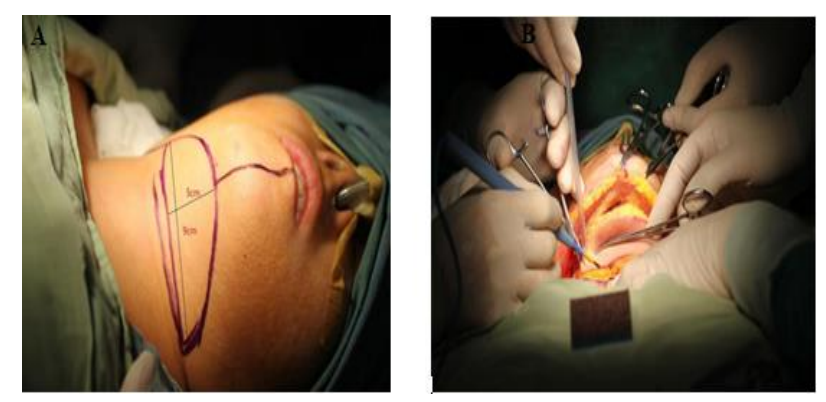

Figures7. (A): Intraoperative design of flap size $5 \times 9 \mathrm{~cm}$; (B): Submental Island Flap raising
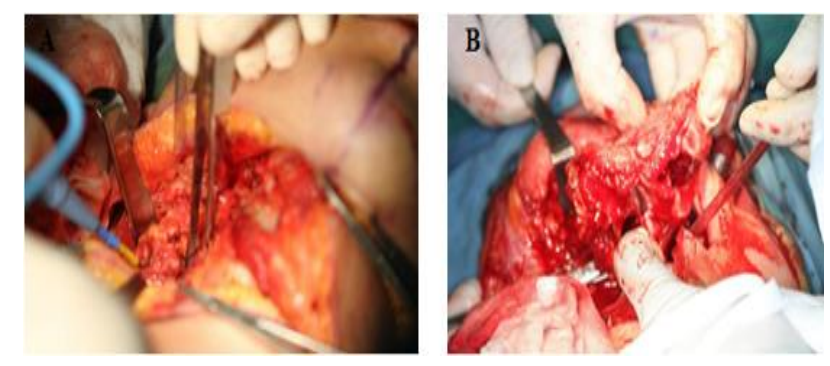

Figures8. (A): Image of neck dissection; (B): Image of Extended resection of the primary tumor through subtotal right maxillectomy and partial left maxillary resection.
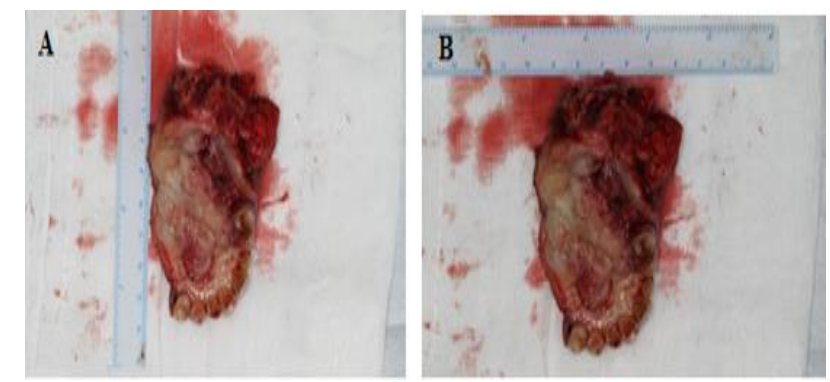

Figures9. (A) and (B): Images of the Resected tumor and Maxilla. 

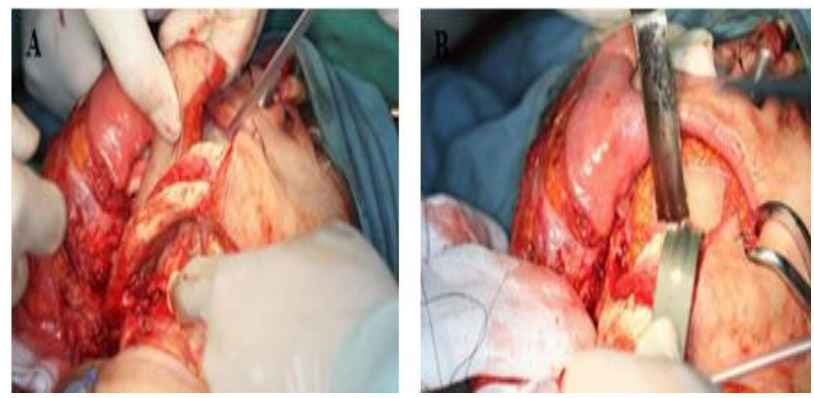

Figures10. (A) and (B): Images of Submental Island Flap transfer and fixation at the recipient site for the reconstruction of the defect
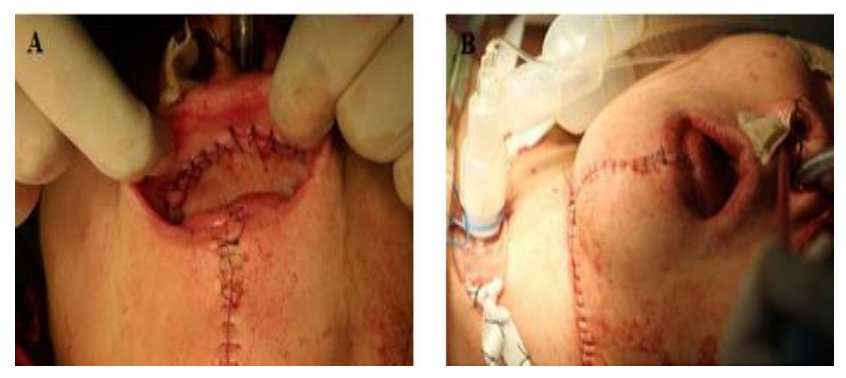

Figures11. (A): Image of the final result of the operation; (B): Image of Tracheostomy.

There is a lack of evidence-based guidelines providing recommendations related to the practice of neck dissection during the treatment of clinically $\mathrm{N}_{0} \mathrm{SCC}$ of palate $[2,5,6,9]$. In many cases, only surgical resection of the primary tumor was carried out without neck dissection. This conservative approach called the "wait and see approach", suggesting close monitoring of the lymph nodes status in the postoperative period, was most practiced in the past years and remains the treatment of choice for some surgeons [2,5]. This could be justified by the fact that the risk of cervical lymph nodes metastases in the SCC of the palate has been considered as low [9,17]. The understanding of this risk has evolved, and the role of neck dissection in clinically $\mathrm{N}_{0}$ neck SCC of palate becomes clearer, with the possibility of occult metastases $[9,17]$. It is widely accepted that neck dissection must be performed if the possibility of neck metastasis exceeds 15 to $20 \%$ $[6,8,9,18]$.

Recently, a probability of occult metastasis over $20 \%$ in SCC of the hard palate, maxillary gingiva, and maxillary alveolus was reported [18]; consequently, many authors recommend performing selective neck dissection during resection of SCC of these sites because of the rate of failing salvage surgery in cases of regional recurrence in cervical lymph nodes after primary tumor resection $[6,8,18]$. The high rate of regional recurrence during the early postoperative period, the limited number of salvageable recurrences, and the poor outcome despite the type of salvage surgery performed represent the 3 main factors that may justify this recommendation of selective neck dissection for the majority of cases of SCC of the palate (all $\mathrm{T}_{2}-\mathrm{T}_{4}$ ) $[5,8,17]$, excepted the case of localized $\mathrm{T}_{1}$ tumors without bone invasion [17]. Usually, an ipsilateral or bilateral supraomohyoidal neck dissection is performed, and improved survival outcomes have been reported [2]. In this case, the selective neck dissection consisted of an ipsilateral supraomohyoidal neck dissection (right levels I, II, III, and Va), and partial contralateral levels I, IIa neck dissection.

The selective neck dissection in the early stage of SCC of the palate has two goals, diagnostic and therapeutic (preventive and curative). It provides important information for correct pathological grading of the tumor, which influences treatment decisions such as a radical neck dissection as a management strategy in case of final pathological assessment reporting positive lymph node metastasis in all the levels involved by selective neck dissection; and the need or not of radiotherapy as adjuvant therapy $[5,8]$.

The maxillary location of SCC represents a clinical variable of local recurrence and regional metastasis [8]. This fact must be taken into account before the ablative surgery for a good choice of reconstructive surgical procedure. Normally, for better functional and esthetic outcomes, the bony defect must be reconstructed by the bony flap, completed by an implant or prosthodontic treatment. But, because of the risk of local recurrence, we decided to delay bony reconstruction after an observational follow-up window of at least 3-4 years. For immediate reconstruction, we used SIF to satisfy the patient's esthetic and functional needs.

The SIF is overlying the main lymph node basin for all oral malignant tumors, especially levels I and II, making its elevation technique difficult and raising the question of its oncological safety. In their study, Elzahaby et al [19] evaluated the adequacy of neck dissection with concomitant SIF raising by examining the final pathological lymph node status after resection of oral SCC; the authors confirmed that SIF does not interfere with neck dissection and it is oncologically safe in patients with $\mathrm{N}_{0}-\mathrm{N}_{1}$ SCC. However, in the case of concomitant SIF raising and neck dissection, the surgeon must pay attention during the neck 
dissection to preserve the blood supply of the submental artery and the venous outflow.

Free flaps remain the primary option for reconstruction of post-ablative oral and maxillofacial defects, but the technique may not be suitable for patients operated previously at the same area, patients with inadequate recipient vessels or vessel-depleted, irradiated neck, elderly patients in poor medical condition, morbid patients who cannot tolerate prolonged anesthesia, and in areas where the extra cost of microvascular surgery limit its access by patients [19-21]. For such cases, pedicled flaps represent the classic option because of their technical simplicity and reliable blood supply [19]. Of all the pedicled flaps, the SIF represents an excellent alternative to free flap for reconstruction palatal and maxillary defects [1].

The advantages provided by the SIF include its reliability, skin quality with good color matching at the recipient area, not bulky, safe, quick and simple to elevate, reliable blood supply with a good arc of rotation, well-hidden donor site scar, low or absence of donor site morbidity [19-21]. Although the SIF represents an excellent reconstructive surgical procedure for palatal and maxillary defects, a thorough evaluation of the submental lymph node basin is recommended either in preoperative or per operative before its raising, transfer, and fixation to the defect site. The presence of lymph node metastasis in either the submental or submandibular regions is a contraindication to the use of SIF. Other contraindications include the prior sacrifice of the submental or facial vascular system, which can occur during excision of the submandibular gland or neck dissection [21].

After resection of the keratinizing SCC of the palate, the remaining maxillary defect was classified as a class $2 b$ according to the first Brown's classification of maxillary defects [22] and class IId according to the new Brown's classification of maxillary defects [23]. In their study, You et al [20] reported that the maxillary defects of class $1,2 \mathrm{a}$ or $3 \mathrm{~b}$ could be reconstructed by the SIF with sizes of skin paddle varying from 4 $\mathrm{x} 12 \mathrm{~cm}$ to $5 \times 12 \mathrm{~cm}$. In this case, the size of the SIF skin paddle was $5 \times 9 \mathrm{~cm}$ (Figure 7A). Because a part of the soft palate was removed during resection of the tumor, this could result in residual soft palate edema. After the flap transfer and fixation at the defect site, the flap volume and soft palate edema could lead to the reduction of intraoral space and affect the patient's breathing due to airway obstruction. To prevent complications secondary to airway obstruction, the tracheostomy was indicated (Figure 11B). The postoperative outcomes were simple. In the study of Eskander et al [1], most patients were downstaged after final pathological assessment, but in the study of Morris et al 2011 [17], most patients were up-staged. After the final pathological assessment of the postoperative specimen (Figure 12), the absence of metastasis in all cervical lymph nodes was confirmed. Thus, the postoperative final pathological TNM-staging was $\mathrm{T}_{4} \mathrm{~N}_{0} \mathrm{M}_{0}$, and the corresponding cancer-staging was classified as Stage IVa. The patient is still being followed up periodically. After 18 months of follow-up, she is doing well, and there is no evidence of recurrence.

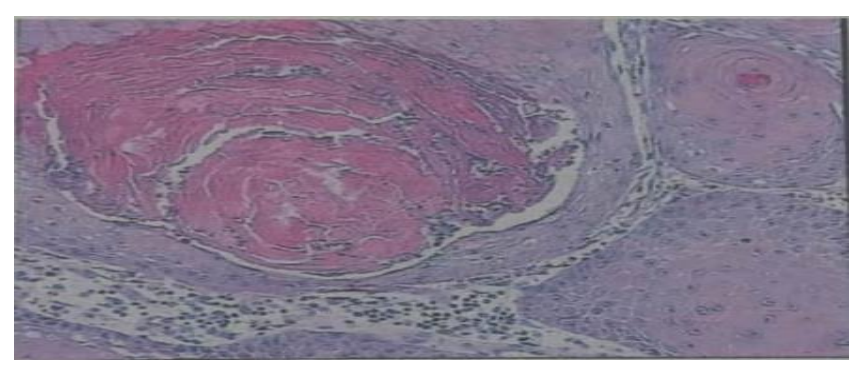

Figure12. Histopathological image of postoperative specimen confirming the diagnosis of Keratinizing squamous cell carcinoma of the right palate (magnification: 40x). Tumor volume was $3 \mathrm{~cm} x 2 \mathrm{~cm} x$ $1.5 \mathrm{~cm}$. Tumor tissue was localized close to bone tissue. No cancer was found in salivary gland tissue. The striated muscle was negative, the cutting edge was negative, and no metastatic carcinoma was found in all the lymph nodes.

\section{Conclusion:}

Keratinizing squamous cell carcinoma of the palate can be seen in a woman of the fourth decade without alcohol drinking and tobacco smoking history. In absence of the common two risk factors of oral squamous cell carcinoma, other risk factors must be researched, such as Hepatitis B virus and local chronic inflammation. An accurate diagnosis requires a combination of clinical, imaging, and histopathological findings. The patient age, tumor size, presence or absence of regional metastases in the cervical lymph nodes, the degree of bone invasion, and the pathological grade of the tumor are important prognosis factors that influence the treatment planning and survival. The present case was a low-grade squamous cell carcinoma (welldifferentiated) with a good prognosis. The treatment strategies of keratinizing squamous cell carcinoma of the palate in clinically negative neck patient include a supraomohyoidal neck dissection, extended resection of the tumor, and reconstruction 
of the defect with the most convenient method according to the case, such as Submental island flap that represented an excellent option for the palatal and maxillary defects in this case. Postoperative outcomes were simple. A periodical follow-up is recommended for early detection of any recurrence.

\section{Acknowledgements and disclosure statements:}

The authors report no conflicts of interest related to this study.

\section{References:}

[1] Eskander A, Strigenz D, Seim N, Ozer E. Submental Artery Island flap with simultaneous level I neck dissection. Head Neck. 2018; 40 (4): 842-845. [Medline: 29331076] [Doi: 10.1002/hed.25044].

[2] Sagheb K, Sagheb K, Taylor KJ, Al-Nawas B, Walter C. Cervical metastases of squamous cell carcinoma of the maxilla: a retrospective study of 25 years. Clin Oral Investig. 2014; 18 (4): 1221-1227. [Medline: 23934238] [Doi: 10.1007/s00784-013-10708].

[3] Cariati P, Cabello-Serrano A, Perez-de Perceval-Tara M, Monsalve-Iglesias F, Martínez-Lara I. Oral and oropharyngeal squamous cell carcinoma in young adults: A retrospective study in Granada University Hospital. Med Oral Patol Oral Cir Bucal. 2017; 22 (6): e679-e685. [Medline: 29053649] [Doi: 10.4317/medoral.21755].

[4] Bijai LK, Mathew P, Jayaraman V, Austin RD. Oral squamous cell carcinoma of palateA case report and review of literature. Int $\mathrm{J}$ Dent Sci Res. 2014; 2 (5): 106-108. [Doi: 10.12691/ijdsr-2-5-1].

[5] Leiser Y, Yudovich K, Barak M, El Naaj I. The Management of Maxillary Squamous Cell Carcinoma-A Retrospective Study. Journal of Cancer Therapy. 2014; 5: 10651071. [doi: 10.4236/jct.2014.512112].

[6] Koshkareva Y, Liu JC, Lango M, Galloway T, Gaughan JP, Ridge JA. Cervical metastasis in squamous cell carcinoma of the hard palate and maxillary alveolus. Ear Nose Throat J. 2016; 95 (10-11): E6-E11. [Medline: 27792826] [Doi: 10.1177/014556131609510-1103].

[7] Singh J. Histopathology of Oral Squamous Cell Carcinoma-A Review. TMU J. Dent. 2014; 1 (4): 141-144.
[8] Montes DM, Carlson ER, Fernandes R, Ghali GE, Lubek J, Ord R, Bell B, Dierks E, Schmidt BL. Oral maxillary squamous carcinoma: an indication for neck dissection in the clinically negative neck. Head Neck. 2011; 33 (11): 1581-5. [Medline: 21990223] [doi: 10.1002/hed.21631].

[9] Joosten MHMA, de Bree R, Van Cann EM. Management of the clinically node negative neck in squamous cell carcinoma of the maxilla. Oral Oncol. 2017; 66: 87-92. [Medline: 28249653] [Doi: 10.1016/j.oraloncology.2016.12.027].

[10] Khan M, Mansoor N, Menon MR, Salam A. Pattern of oral squamous cell carcinoma-A study. Pakistan Oral and Dental Journal. 2008; 27 (2): 277-282.

[11] Foy JP, Bertolus C, Boutolleau D, Agut H, Gessain A, Herceg Z, Saintigny P. Arguments to Support a Viral Origin of Oral Squamous Cell Carcinoma in Non-Smoker and Non-Drinker Patients. Front Oncol. 2020; 10: 822. [Medline: 32528893] [Doi: 10.3389/fonc.2020.00822].

[12] Komori MF, Kimura T, Kariya S, Onoda T, Takeda S, Mizukawa N, Iida S, Kimata Y, Nishizaki K. Epidemiological Correlations Between Head and Neck Cancer and Hepatitis B Core Antibody Positivity. Anticancer Res. 2020; 40 (4): 2393-2403. [Medline: 32234943] [Doi: 10.21873/anticanres.14209].

[13] Dona S, Borsetto D, Fussey J, Biscaro V, Vian E, Spinato G, Menegaldo A, Da Mosto MC, Rigoli R, Polesel J, Boscolo-Rizzo P. Association between hepatitis $\mathrm{C}$ and $\mathrm{B}$ viruses and head and neck squamous cell carcinoma. J Clin Virol. 2019; 121: 104209. [Medline: 31711028] [Doi: 10.1016/j.jcv.2019.104209].

[14] Song C, Li J, Liu Y, Chen JG, Ge Z, Zhu J, Dai J, Du LB, Yu C, Guo Y, Bian Z, Yang L, Chen Y, Chen Z, Liu J, Jiang J, Zhu L, Zhai X, Jiang Y, Ma H, Jin G, Shen H, Li L, Hu Z; China Kadoorie Biobank Collaborative Group. Associations Between Hepatitis B Virus Infection and Risk of All Cancer Types. JAMA Netw Open. 2019; 2 (6): e195718. [Medline: 31199446] [Doi: 10.1001/jamanetworkopen.2019.5718].

[15] Kocoglu H, Karaca M, Tural D, Hocaoglu E, Okuturlar Y, Fetullahoglu Z, Gunaldi M, Ciftci R, Tuna S, Yucil OK, Yuce OK, Ozet 
G, Ozet A, Benekli M. Hepatitis B and C rates are significantly increased in certain solid tumors: A large retrospective study. J Cancer Res Ther. 2018; 14: S774-S778. [Medline: 30249902] [Doi: 10.4103/09731482.174544].

[16] Shah JP, Gil Z. Current concepts in management of oral cancer--surgery. Oral Oncol. 2009; 45 (4-5): 394-401. [Medline: 18674952]

[Doi:

10.1016/j.oraloncology.2008.05.017].

[17] Morris LG, Patel SG, Shah JP, Ganly I. High rates of regional failure in squamous cell carcinoma of the hard palate and maxillary alveolus. Head Neck. 2011; 33 (6): 824-30. [Medline: 20949448]

[Doi: 10.1002/hed.21547].

[18] Park HJ. Is elective neck dissection needed in clinically NO neck in maxillary cancer? $\mathrm{J}$ Korean Assoc Oral Maxillofac Surg. 2014; 40 (3): 101-2. [Medline: 25045635] [Doi: 10.5125/jkaoms.2014.40.3.101].

[19] Elzahaby IA, Roshdy S, Shahatto F, Hussein $\mathrm{O}$. The adequacy of lymph node harvest in concomitant neck block dissection and submental island flap reconstruction for oral squamous cell carcinoma; a case series from a single Egyptian institution. BMC Oral Health. 2015; 15: 80. [Medline: 26168787] [Doi: 10.1186/s12903-015-0064-0].
[20] You YH, Chen WL, Wang YP, Liang J, Zhang DM. Reverse facial-submental artery island flap for the reconstruction of maxillary defects after cancer ablation. J Craniofac Surg. 2009; 20 (6): 2217-20. [Medline: 19934677]

[Doi: 10.1097/SCS.0b013e3181bf84d7].

[21] Genden EM, Buchbinder D, Urken ML. The submental island flap for palatal reconstruction: a novel technique. J Oral Maxillofac Surg. 2004; 62 (3): 387-90. [Medline: 15015176] [Doi: 10.1016/j.joms.2003.06.009].

[22] Brown JS, Rogers SN, McNally DN, Boyle M. A modified classification for the maxillectomy defect. Head Neck. 2000; 22 (1): 17-26. [Medline: 10585601] [Doi: 10.1002/(sici)1097-0347(200001)22:1<17: aid-hed4>3.0.co;2-2].

[23] Brown JS, Shaw RJ. Reconstruction of the maxilla and midface: introducing a new classification. Lancet Oncol. 2010; 11 (10): 1001-8. [Medline: 20932492] [Doi: 10.1016/S1470-2045(10)70113-3]. 